Somnologie 2010 · 14:70-72

DOI 10.1007/s11818-010-0448-y

Online publiziert: 5. März 2010

(c) Springer-Verlag 2010

\author{
G. Mayer \\ c/o Hephata-Klinik, Schwalmstadt
}

\title{
Zum Tode von Prof. Dr. med. Dr. rer. nat. Jörg Hermann Peter
}

sere Wege begleitet und mit geprägt. Viele von uns hat er durch schwierige berufliche und persönliche Situationen begleitet. Er hat immer wieder ermutigt, den eigenen Weg zu finden und zu gehen.

Prof. Peter wurde am 18. Februar 1945 in Treisbach/Wetter geboren. In Marburg studierte er Humanmedizin und Psychologie, beides schloss er mit einer Promotion ab. Im Rahmen seiner Promotionsarbeit im Fach Psychologie beschäftigte er sich bei Lokomotivführern mit den Zusammenhängen zwischen Vigilanz, Schläfrigkeit, Leistungsfähigkeit und Veränderungen im autonomen System. Berufliche Stationen waren dabei das Physiologische Institut und das Institut für medizinisch-biologische Statistik und Dokumentation der Philipps-Universität Marburg. 1979 wechselte er zur Weiterbildung zum Facharzt für Innere Medizin in die Medizinische Poliklinik. Dort beschäftigte er sich ab 1981 mit einem „neuen“ Phänomen in der Medizin, der Schlafapnoe. Er begann mit dem Aufbau eines Schlaflabors, zunächst „Arbeitsgruppe Klinische Zeitreihenanalyse“ genannt, zur Untersuchung der physiologischen und pathophysiologischen Phänomene der Atmungsregulation und der Herz-Kreislauf-Funktionen im Schlaf. Seine Kenntnisse in Psychophysiologie, Methodik und Medizin prädestinierten ihn dazu, auf diesem Gebiet Pionierarbeit zu leisten und die vielfältigen, überwiegend ungeklärten Aspekte dieses Störungskomplexes zu untersuchen und in einen Gesamtkontext stellen. 1986 habilitierte er sich mit dem Thema „Die Erfassung der Schlafapnoe in der Inneren Medizin“. Eine Reihe von Inno- vationen, die auf dem Gebiet später in die Medizin eingeführt wurden, hat hier ihren Ausgang genommen.

Unter seiner Federführung entstand das weltweit erste ambulant einsetzbare 4-Kanal-Messgerät, der „Marburger Koffer", sowie stationär einfach anwendbare 8- und Mehr-Kanal-Systeme mit denen eine zeitgerechte Versorgung der immer größeren Zahl an Patienten mit entsprechenden Diagnosen möglich wurde. Hierdurch brachte er früh ambulante Methoden in der Schlafmedizin zum Einsatz, deren eingeschränkte Aussagekraft er aber stets berücksichtigt wissen wollte. Wissenschaftlich eröffnete sich mit dem Einsatz der Polygraphen die Möglichkeit zu epidemiologischen Untersuchungen, die Prof. Peter bei internistischen Patienten und hier insbesondere bei Patienten mit Herz-Kreislauf-Erkrankungen durchführte. So entstand als Ergebnis seiner damaligen Arbeit bereits Anfang der 1980er-Jahre das Konzept des Risikofaktors der obstruktiven Schlafapnoe für den arteriellen Hypertonus, für die koronare Herzkrankheit sowie für Patienten mit schwerer Herzinsuffizienz. 1985 führte er die nasale Beatmungstherapie für $\mathrm{Pa}$ tienten mit obstruktiver Schlafapnoe zu einem Zeitpunkt in Deutschland ein, als diese Therapie international noch kontrovers diskutiert wurde.

Seine Fähigkeit, wissenschaftliche und politische Visionen zu entwickeln, hat ihn zu einem der führenden Köpfe der Schlafmedizin gemacht. Für Zuhörer war es immer wieder überraschend, wie scheinbar Unzusammenhängendes absolut systematisch wurde. Diese Fähigkeit hat er ganz 
entscheidend auch politisch eingesetzt. Durch viele Gespräche mit Kollegen aller Fachrichtungen hat er eine Spaltung der Schlafmedizin in eine Medizin einzelner Fachrichtungen verhindert. 1991 war er Gründungsmitglied der DGSM.

Unermüdlich hat er darauf hingewiesen, dass eine ,junge Wissenschaft“ auch gelehrt werden muss und einen gebührenden Platz im Gesundheitssystem einnehmen sollte. Er hat Anfang der 199oerJahre ein Curriculum für die Schlafmedizin entscheidend mitentwickelt. Als ersten Schritt zu einer formalen Anerkennung für Schlafmediziner hat er den „Qualifikationsnachweis Schlafmedizin“ der DGSM mitbegründet. Er hat unsere Fachzeitschrift "Somnologie“ mit aus der Taufe gehoben und damit den Schritt zur Aufnahme in die Arbeitsgemeinschaft Wissenschaftlicher Medizinischer Fachgesellschaften (AWMF) ermöglicht. Als weitere Anstrengungen für die Anerkennung der Schlafmedizin seien hier nur die Verhandlungen mit den Ärztekammern um die Aufnahme schlafmedizinischer Leistungen in den Katalog der ärztlichen Gebührenordnung genannt, das Erstellen eines Weißbuchs Schlafmedizin und einer S2-Leitlinie „Nichterholsamer Schlaf“. Wenn Deutschland heute in Europa mit seiner Ausbildung, seiner Struktur und der „Zusatzbezeichnung Schlafmedizin“ ganz vorn steht, ist das nicht unwesentlich Prof. Peters Verdienst.

Die Fähigkeit, Forschung und Wissenschaft insbesondere auch unter dem Versorgungsaspekt erfolgreich durchzuführen und immer wieder mit neuen Impulsen zu versehen, hat Prof. Peter weit über die Nation hinaus auch internationale Anerkennung eingebracht. Als Vertreter der Schlafmedizin und nicht primär als Vertreter seines Fachgebietes hat er in nationalen und internationalen Kongressen immer die gesamte Schlafmedizin in ihrer Interdisziplinarität vertreten. Er hat dadurch auch europaweit in vielen Ländern eine Aufsplitterung der Schlafmedizin verhindern können.

Sein erfolgreiches Beschreiten von wissenschaftlichem Neuland brachte nicht immer Anerkennung mit sich, häufig sah er sich auch Unverständnis und Widerständen ausgesetzt. Diese Erfahrungen waren teilweise bitter, haben ihn aber auch geprägt. So hat er seine Vorstellungen und Überzeugungen immer klar, konsequent und unbestechlich vertreten. Diejenigen, die sich von seiner Art „überfahren“ fühlten, mussten ihm später oft Recht geben. Sein Handeln war aber nicht nur geprägt durch Geradlinigkeit und Direktheit, sondern - wenn es darum ging, übergeordnete Ziele durchzusetzen - auch durch Bescheidenheit, die ihn seine eigenen Belange zurückstellen ließ. Wir glauben, dass diese Mischung aus Vision, Geradlinigkeit, Pragmatismus und Bescheidenheit den Menschen Hermann Peter ausgemacht haben. Lehrer prägen die Denkweise und das Handeln ihrer Schüler. Sie sind gute Lehrer, wenn sie nicht nur Wissen vermitteln, sondern auch Haltungen, Moral und Lösungsstrategien. Wir wissen, dass viele Schlafmediziner in den vergangenen 30 Jahren bei Prof. Peter etwas von diesen Eigenschaften mitnehmen konnten, sodass wir meinen, ihn in die Tradition großer medizinischer Lehrer einreihen zu dürfen.

Seine langjährige Krankheit hat ihn früh aus unserer vordersten Reihe gerissen. Er ist uns aber immer ein Vordenker und Mitdenker geblieben. Mit der „Enzyklopädie der Schlafmedizin“ hat er noch einmal ein Anliegen verwirklichen können, die Erstellung eines umfassenden Standardwerkes. In diesem Sinne hat er auch an der S3-Leitlinie „Nichterholsamer Schlaf/Schlafstörungen“ bis zum Schluss aktiv mitgewirkt. Ein wirklich volles Leben, an dem wir über viele Jahre teilnehmen durften.

Die DGSM verabschiedet sich in tiefer Trauer und wird Prof. Jörg Hermann Peter ein ehrendes und dankbares Gedenken bewahren.

Wir trauern um den Verlust eines Freundes, Lehrers und großen Vorbilds als Mensch und Wissenschaftler.

Prof. Dr. Heinrich Becker

Prof. Dr. Geert Mayer

Prof. Dr. Thomas Penzel

Prof. Dr. Thomas Podszus

\section{Korrespondenzadresse}

Prof. Dr. G. Mayer

c/o Hephata-Klinik

Schimmelpfengstraße 2, 34613 Schwalmstadt

dgsm.mayer@t-online.de 
Hier steht eine Anzeige.

黛 Springer 